\title{
Task shifting in the management of hypertension in Kinshasa, Democratic Republic of Congo: a cross-sectional study
}

Aimée M. Lulebo ${ }^{1 *}$, Didine K. Kaba ${ }^{1}$, Silvestre E.-H. Atake², Mala A. Mapatano ${ }^{1}$, Eric M. Mafuta', Julien M. Mampunza ${ }^{3}$ and Yves Coppieters ${ }^{4}$

\begin{abstract}
Background: The Democratic Republic of the Congo (DRC) is characterized by a high prevalence of hypertension (HTN) and a high proportion of uncontrolled HTN, which is indicative of poor HTN management. Effective management of HTN in the African region is challenging due to limited resources, particularly human resources for health. To address the shortage of health workers, the World Health Organization (WHO) recommends task shifting for better disease management and treatment. Although task shifting from doctors to nurses is being implemented in the DRC, there are no studies, to the best of our knowledge, that document the association between task shifting and HTN control. The aim of this study was to investigate the association between task shifting and HTN control in Kinshasa, DRC.
\end{abstract}

Methods: We conducted a cross-sectional study in Kinshasa from December 2015 to January 2016 in five general referral hospitals (GRHs) and nine health centers (HCs). A total of 260 hypertensive patients participated in the study. Sociodemographic, clinical, health care costs and perceived health care quality assessment data were collected using a structured questionnaire. To examine the association between task shifting and HTN control, we assessed differences between GRH and HC patients using bivariate and multivariate analyses.

Results: Almost half the patients were female (53.1\%), patients' mean age was $59.5 \pm 11.4$ years. Over threefourths of patients had uncontrolled HTN. There was no significant difference in the proportion of GRH and HC patients with uncontrolled HTN (76.2\% vs $77.7 \%, p=0.771)$. Uncontrolled HTN was associated with co-morbidity $(\mathrm{OR}=10.3 ; 95 \% \mathrm{Cl}: 3.8-28.3)$ and the type of antihypertensive drug used (OR $=4.6 ; 95 \% \mathrm{Cl}: 1.3-16.1)$. The mean healthcare costs in the GRHs were significantly higher than costs in the HCS (US\$ $34.2 \pm$ US\$3.34 versus US\$ 7. $7 \pm$ US\$ 0.6, respectively).

Conclusion: Uncontrolled HTN was not associated with the type of health facility. This finding suggests that the management of HTN at primary healthcare level might be just as effective as at secondary level. However, the high proportion of patients with uncontrolled HTN underscores the need for HTN management guidelines at all healthcare levels.

Keywords: Hypertension management, Task shifting, Democratic Republic of Congo

\footnotetext{
* Correspondence: aimelulebo@yahoo.fr

${ }^{1}$ Kinshasa School of Public Health, Faculty of Medicine, University of Kinshasa,

Kinshasa, Democratic Republic of the Congo

Full list of author information is available at the end of the article
} 


\section{Background}

In 2010, 34.5 million people died from non-communicable diseases (NCDs). These deaths accounted for more than two thirds of the deaths worldwide [1]. Further, nearly $80 \%$ of these deaths occurred in low- and middle-income countries (LMICs) [2]. Hypertension (HTN) is among the main risk factors of NCDs. In 2008, the estimated prevalence of HTN in Africa was $46 \%$, which translates to a population of nearly 30 million adults [3, 4]. Between 2003 and 2009, the WHO STEP wise approach to Surveillance (STEPS) study that was carried out in 20 African countries found that the prevalence of HTN ranged from $19.3 \%$ to $39.6 \%$ [5]. In the Democratic Republic of Congo (DRC), the prevalence of HTN was $32.1 \%$ in men and $31.5 \%$ in women in 2014 [6].

Sub-Saharan African (SSA) countries are experiencing one of the most rapid epidemiological transitions but their health systems are more oriented to the management of infectious diseases [7-9]. Effective management of HTN in SSA is challenging due to limited resources, particularly human resources for health [10, 11]. In 2009, for example, it was estimated that there were 2 physicians and 11 nurses/midwives available per 10,000 populations in SSA compared with 19 physicians and 49 nurses/midwives per 10,000 populations in North America. In 2009, in the Democratic Republic of Congo (DRC), the total number of physicians was estimated at 5827 ( 1 physician per 10,000 population) and the total number of nurses at 28,789 ( 5 nurses per 10,000 population) [12].

Task shifting has been identified as a means to address the health worker crisis and improve access and costeffectiveness in health systems [13]. Task shifting, which dates back to the 1970s-1980s when auxiliary nurses took up the health care provider role, describes a strategy where some tasks that are normally performed by a physician or other specialized health staff are assigned to a health professional with a different or lower level of education and training, or to a person specifically trained to perform a limited task only, without having formal health education $[14,15]$. Task shifting is a viable strategy in LMICs for the primary and secondary prevention of NCDs [14]. Previous studies have shown that nurses are cheaper to employ and train than doctors, Furthermore, nurses are one of the largest groups of qualified health care providers [16]. Task shifting has been shown to be cost-effective $[17,18]$.

Task shifting in the DRC, is based on the Primary Health Care (PHC) model. The health center (HC), which is managed by a head nurse, is the first contact between patients and health system. HCs provide accessible and affordable primary health care. Patients requiring further care are referred from the $\mathrm{HC}$ to a General Referral Hospital (GRH) [19]. Although a recent study carried out in HCs of Kinshasa found a low proportion of patients with controlled HTN [20], to the best of our knowledge, there have been no studies that have examined the association between task shifting and the management of HTN in the DRC. The aim of this study was to investigate the association between task shifting and the management of HTN by comparing HTN control in HCs and GRHs patients.

\section{Methods}

Study design

We conducted a cross-sectional study in Kinshasa Primary Health-Care (KPHC) network facilities from December 2015 to January 2016. The KPHC network consists of 51 facilities (11 GRHs and $40 \mathrm{HCs}$ ) that serve patients living in Kinshasa [21].

\section{Study population}

The study included patients with an HTN diagnosis made by a healthcare provider and who were receiving care in the KPHC network facilities. Patients were eligible for the study if they were aged 18 years and older, presented for care in a participating health facilities on the day of the survey, and agreed to participate in the study.

\section{Sampling}

The sample size was computed using the following formula:

$$
n \geq \frac{[p 1(1-p 1)+p 2(1-p 2)]}{(P 1-P 2)} x(z 1-\alpha+z 1-\beta) 2
$$

Where $\mathrm{p} 1$ represents the proportion of patients with controlled HTN at the HC level (15.6\%) [19]; p2 represents the proportion of patients with controlled HTN in the GRH level (we assumed that the proportion would be twice that in HCs (31.2\%); $\mathrm{z}$ is the value of the standard normal distribution corresponding to a significance level of alpha of 0.05 (1.96); and $\beta$ is power (80\%), z1$\beta=0.84$.

The minimal sample size computed was 112 patients per group or 224 patients for both groups. To recruit patients, we obtained the list of GRHs and HCs from the KPHC network and visited all them to know the dates when HTN patients have follow-up clinics. We then visited the facilities on clinic dates and successively recruited patients until the desired sample size was achieved. The minimum sample size was achieved after visiting nine $\mathrm{HCs}$ and five GRHs.

\section{Data collection}

Five trained data collectors' conducted face-to-face interviews using a structured questionnaire. The questionnaire 
was pretested and translated into the local language (Lingala) before data collection. The questionnaire elicited information on the following variables: patients' socio-demographic (sex, age, marital status, educational level, income) and clinical characteristics (duration of HTN, co-morbidity); health system variables (patient-provider relationship, the time devoted to consultation, waiting time, affordability and availability of healthcare); treatment related variables (types of antihypertensive drugs and dosage, experience of medication side effects, treatment adherence) and medical-related costs (consultation fees, medicines, laboratory, transportation and food).

\section{Study variables}

The dependent variable was uncontrolled HTN, which was defined as having a systolic blood pressure (SBP) $\geq 140 \mathrm{mmHg}$ and/or a diastolic blood pressure (DBP) $\geq 90 \mathrm{mmHg}$ for patients without co-morbidity or having $\mathrm{SBP} \geq 130 \mathrm{mmHg}$ and/or $\mathrm{DBP} \geq 80 \mathrm{mmHg}$ for patients with co-morbidity [22]. The primary explanatory variable, task shifting, was defined as the management of HTN at HCs level.

Co-morbidity was defined as HTN associated with diabetes mellitus or target organ damage (heart disease, stroke, or chronic kidney disease) based on diagnosis made by a healthcare provider. Antihypertensive types were categorized in two groups, monotherapy if a patient was on one type of antihypertensive medication and multitherapy if a patient was on two or more types of antihypertensive medications. Treatment adherence was measured using the Morisky Scale, which is a validated four-item scale with good internal consistency based on studies of inner-city patients with HTN [23, 24]. Patients responded "yes" or "no" to four questions (Do you ever forget to take your medicine? Are you careless at times about taking your medicine? When you feel better, do you sometimes stop taking your medicine? Sometimes if you feel worse when you take the medicine, do you stop taking it?). Based on their responses, patients were categorized into three groups: high, medium and low adherence. To facilitate statistical analysis, adherence was dichotomized with patients with low or medium adherence classified as non-adherent and patients with high adherence classified as adherent [25].

The costs of medicines were reported on a monthly basis. The transportation costs comprised roundtrip travel costs incurred by the patient and anyone who accompanied them to the health facility for HTN-related care. Food costs comprised expenditures related to food during HTN-related health facility visits. Consultation, medicines and laboratory costs were obtained from patient record notebooks, invoices and bills provided by patients. All KPHC patients have a patient record notebook where all information related to their care including costs are recorded. Transportation and food related costs were self-reported. The cost computations did not include productivity and opportunity costs. Costs were provided in Congolese Democratic Francs (CDF), the local currency, and converted into United States Dollars (US\$) using the average exchange rate during the study period (920 CDF to US\$ 1). To account for inflation, a rate of $2 \%$ was used in computing all costs incurred outside the reference period (2015 to 2017).

\section{Statistical analysis}

Data were entered into Epi data then exported to SPSS (Statistical Package for Social Sciences) version 20.0 (SPSS, Inc., Chicago, IL, USA) and Microsoft Excel for analysis. Descriptive statistics were used to summarize patients' characteristics. Categorical variables were reported as frequencies and percentages. Continuous variables were reported using means with standard deviation. The Student's $t$ test and $x^{2}$ test were used to compare means and proportions respectively. Logistic regression was used to examine the association between task shifting and uncontrolled HTN after adjusting for others factors like sociodemographic, clinical factors. For all analyses, a $p$-value of less than 0.05 was considered statistically significant.

\section{Ethical considerations}

The study protocol was reviewed and approved by the institutional review board of the Kinshasa School of Public Health. All study participants provided written informed consent.

\section{Results}

Patients' socio-demographic and clinical characteristics

Patients' socio-demographic and clinical characteristics are summarized in Table 1. Almost half of the patients were female (53.1\%). The patients' mean age was 59.5 years (standard deviation $=11.4$ years). $\mathrm{A}$ significantly higher proportion of GRH patients than $\mathrm{HC}$ patients had ever attended school $(87.7 \%$ vs $77.7 \%, p=0.035)$. Co-morbidity was significantly more common in GRH patients compared with $\mathrm{HC}$ patients $(45.4 \%$ vs $25.4 \%, p<0.001)$. The majority (94.2\%) of patients had been diagnosed with HTN within the 5 years preceding the study. Only $23 \%$ of all patients had controlled HTN. The proportion of patients with uncontrolled HTN did not differ by type of health facility $(76.2 \%$ among GRH patients vs $77.7 \%$ among $\mathrm{HC}$ patients, $p=0.771$ ).

\section{Quality of health care}

Patients' assessments of the quality of care are summarized in Table 2. A significantly greater proportion of GRH patients than HC patients stated that the consultation time was sufficient $(96.9 \%$ vs $81.5 \%, p<0.001)$. No 
Table 1 Patients' socio demographic and clinical characteristics, by health facility

\begin{tabular}{|c|c|c|c|c|}
\hline Variables & $\begin{array}{l}\text { GRH patients } \\
(\%) \\
n=130\end{array}$ & $\begin{array}{l}\text { HC patients } \\
(\%) \\
n=130\end{array}$ & $\begin{array}{l}\text { Total } \\
(\%) \\
n=260\end{array}$ & $p$-value \\
\hline \multicolumn{5}{|l|}{ Gender } \\
\hline Male & 46.9 & 46.9 & 46.9 & 0.999 \\
\hline Female & 53.1 & 53.1 & 53.1 & \\
\hline \multicolumn{5}{|l|}{ Attended school } \\
\hline Yes & 87.7 & 77.7 & 82.7 & 0.035 \\
\hline No & 12.3 & 22.3 & 17.3 & \\
\hline \multicolumn{5}{|l|}{ Has a source of income } \\
\hline Yes & 60.8 & 66.2 & 63.5 & 0.371 \\
\hline No & 39.2 & 33.8 & 36.5 & \\
\hline \multicolumn{5}{|l|}{ Marital status } \\
\hline Married/cohabiting & 72.3 & 67.7 & 70.0 & 0.421 \\
\hline $\begin{array}{l}\text { Single/separated/ } \\
\text { divorced /widowed }\end{array}$ & 27.7 & 32.3 & 30.0 & \\
\hline \multicolumn{5}{|l|}{ Co-morbidity } \\
\hline Yes & 45.4 & 25.4 & 35.4 & $<0.001$ \\
\hline No & 54.6 & 74.6 & 64.6 & \\
\hline \multicolumn{5}{|l|}{ HTN duration } \\
\hline$<5$ & 94.6 & 93.8 & 94.2 & 0.798 \\
\hline$\geq 5$ & 5.4 & 6.2 & 5.8 & \\
\hline \multicolumn{5}{|l|}{ HTN control } \\
\hline Yes & 23.8 & 22.3 & 23.1 & 0.771 \\
\hline No & 76.2 & 77.7 & 76.9 & \\
\hline Mean age \pm SD & $58.0 \pm 11.6$ & $61.05 \pm 11.0$ & $59.5 \pm 11.4$ & \\
\hline$[95 \% \mathrm{Cl}]$ & {$[55.9 ; 60.1]$} & {$[59.1 ; 62.9]$} & {$[58.1 ; 60.9]$} & \\
\hline
\end{tabular}

Cl confidence interval, GRH General Referral Hospital, HC Health Center, SD standard deviation

statistically significant differences between GRH and HC patients were observed in terms of perceived accessibility, waiting times, affordability, satisfaction with the relationship with healthcare providers, types of antihypertensive drugs used, adherence to medication, and control of HTN. A greater proportion of GRH patients than HC patients reported medication side effects $(22.6 \%$ vs $8.3 \%$, $p=0.003)$. The proportion of patients who were not adherent to medication did not differ by facility (GRH $47.2 \%$ versus $\mathrm{HC} 38.5 \%, p=0.204)$. No significant difference was also found between the two groups with regard to the control of HTN.

\section{Correlates of uncontrolled HTN}

Results of the logistic regression model employed to assess the factors associated with HTN control are summarized in Table 3. The type of facility was not significantly associated with HTN control. Comorbidity and the type of antihypertensive medication
Table 2 Quality of health care assessment

\begin{tabular}{|c|c|c|c|c|}
\hline Variables & $\begin{array}{l}\text { GRH patients } \\
(\%) \\
n=130\end{array}$ & $\begin{array}{l}\text { HC patients } \\
(\%) \\
n=130\end{array}$ & $\begin{array}{l}\text { Total } \\
(\%) \\
N=260\end{array}$ & $p$-value \\
\hline \multicolumn{5}{|l|}{ Time given by HCP } \\
\hline Sufficient & 96.9 & 81.5 & 89.2 & $<0.001$ \\
\hline Insufficient & 3.10 & 18.5 & 10.8 & \\
\hline \multicolumn{5}{|l|}{ Distance HCF-Home } \\
\hline$<5 \mathrm{~km}$ & 59.2 & 66.2 & 62.7 & 0.252 \\
\hline$\geq 5 \mathrm{~km}$ & 40.8 & 33.8 & 37.3 & \\
\hline \multicolumn{5}{|l|}{ Waiting time } \\
\hline$\leq 30 \min$ & 32.3 & 36.9 & 34.6 & 0.438 \\
\hline$>30 \mathrm{~min}$ & 67.7 & 63.1 & 65.4 & \\
\hline \multicolumn{5}{|c|}{ Perception of treatment cost } \\
\hline Unaffordable & 63.8 & 70.0 & 66.9 & 0.296 \\
\hline Affordable & 36.2 & 30.0 & 33.1 & \\
\hline \multicolumn{5}{|l|}{ Perception of relationship } \\
\hline $\begin{array}{l}\text { Very good(excellent)/ } \\
\text { good }\end{array}$ & 96.9 & 92.3 & 94.6 & 0.109 \\
\hline Somewhat good/bad & 3.1 & 7.7 & 5.4 & \\
\hline \multicolumn{5}{|l|}{ Type of anti-hypertensive } \\
\hline Multitherapy & 11.5 & 23.8 & 17.7 & 0.046 \\
\hline Monotherapy & 70.0 & 60.0 & 65.0 & 0.951 \\
\hline No treatment & 18.5 & 16.2 & 17.3 & \\
\hline \multicolumn{5}{|l|}{ Experience of side effects } \\
\hline Yes & 22.6 & 8.3 & 15.3 & 0.003 \\
\hline No & 77.4 & 91.7 & 84.7 & \\
\hline \multicolumn{5}{|l|}{ Treatment adherence } \\
\hline Yes & 52.8 & 61.5 & 57.2 & 0.204 \\
\hline No & 47.2 & 38.5 & 42.8 & \\
\hline
\end{tabular}

GRH General Referral Hospital, $H C$ Health Center, HCP health care provider, HCF health care facility

used were associated with HTN control. Patients with co-morbidity were more likely than those without comorbidity to have uncontrolled HTN (adjusted $\mathrm{OR}=10.3$; 95\% CI: 3.8-28.3) while patients on multiple medications were more likely than those on a single medication to have uncontrolled HTN (adjusted $\mathrm{OR}=4.6$; $95 \% \mathrm{CI}: 1.3-16.1)$.

\section{Cost analysis}

Table 4 shows the results of the cost analysis. In the $\mathrm{HC}$, the most expensive costs were medication costs (US\$4.4 [95\% CI: US\$3.4 - US\$5.4]) while in the GRH the most expensive costs were laboratory costs (US\$23.6 [95\% CI: US\$ 16.9 - US\$ 30.3]).The mean total cost was lower in the HC than in the GRH (US\$ 7.7 [95\% CI: US\$ 6.6 - US\$ 8.8 ] versus US\$34.2 [95\% CI: US\$27.6 - US\$40.9]). 
Table 3 Bivariate and multivariate analysis of factors associated with uncontrolled HTN

\begin{tabular}{|c|c|c|c|c|}
\hline Variables & $\begin{array}{l}\text { Crude OR } \\
{[95 \% \mathrm{Cl}]}\end{array}$ & $p$-value & Adjusted OR $[95 \% \mathrm{CI}]^{\mathrm{a}}$ & $p$-value \\
\hline $\begin{array}{l}\text { Gender } \\
\text { (male vs female) }\end{array}$ & $1.3[0.7-2.4]$ & 0.353 & $1.3[0.6-2.8]$ & 0.430 \\
\hline \multicolumn{5}{|l|}{ Attended school } \\
\hline No & $0.8[0.4-1.6]$ & 0.530 & $1.7[0.5-5.2]$ & 0.365 \\
\hline Yes & 1 & & & \\
\hline \multicolumn{5}{|l|}{ Co-morbidity } \\
\hline Yes & $8.5[3.3-22.1]$ & $0.000^{b}$ & $10.3[3.8-28.3]$ & $0.000^{\mathrm{b}}$ \\
\hline No & 1 & & 1 & \\
\hline \multicolumn{5}{|c|}{ Type of anti-hypertensive drugs } \\
\hline Multitherapy & $4.9[1.4-16.6]$ & $0.011^{b}$ & $4.6[1.3-16.1]$ & $0.017^{b}$ \\
\hline No treatment & $0.8[0.4-1.6]$ & 0.446 & $0.6[0.3-1.3]$ & 0.199 \\
\hline Monotherapy & 1 & & 1 & \\
\hline \multicolumn{5}{|c|}{ Experience of side effects } \\
\hline Yes & $1.0[0.4-2.5]$ & 0.965 & $1.1[0.4-3.1]$ & 0.786 \\
\hline No & 1 & & & \\
\hline \multicolumn{5}{|c|}{ Treatment adherence } \\
\hline No & $0.9[0.4-1.6]$ & 0.658 & $0.9[0.4-1.7]$ & 0.658 \\
\hline Yes & 1 & & & \\
\hline \multicolumn{5}{|l|}{ Time given by HCP } \\
\hline Insufficient & $1.1[0.4-2.9]$ & 0.827 & $0.6[0.2-2.0]$ & 0.390 \\
\hline Sufficient & 1 & & & \\
\hline \multicolumn{5}{|l|}{ Type of HCF } \\
\hline $\mathrm{HC}$ & $1.1[0.6-1.9]$ & 0.768 & $0.6[0.3-1.2]$ & 0.160 \\
\hline GRH & 1 & & 1 & \\
\hline
\end{tabular}

$\mathrm{Cl}$ confidence interval, GRH general referral hospital, $H C$ health center, $H C P$ health care provider, HCF health care facility, OR odds ratio

${ }^{a}$ Adjusted for gender, having attended school, the time given/allocated by the provider, the experience of side effects, types of anti-hypertensive, treatment adherence and co-morbidity; ${ }^{\mathrm{b}}$ statistically significant

\section{Discussion}

In this study we investigated the association between task shifting and HTN control. Specifically, we compared patients seen in primary health centers that are managed by a head nurse and patients attending general referral hospitals who are typically managed by physicians. Similar to other studies, we found that majority of patients $(76.9 \%)$ in both types of facilities had uncontrolled HTN $[20,26]$. Uncontrolled HTN was not associated with the type of health facility. In contrast, Fahey and colleagues, who conducted a systematic review of randomized controlled trials (RCTs) for management of HTN, found that patients had a greater reduction in blood pressure when followed-up by non-physician health professionals [27]. However, unlike our study, Fahey's study was based on RCTs where non-physicians were trained on management of HTN. Fahey's study also assessed more organizational and structural factors associated with HTN control than our study.
Table 4 Analysis of healthcare costs in United States Dollars (US\$), by facility type

\begin{tabular}{lll}
\hline Healthcare costs & $\begin{array}{l}\text { General Referral Hospital } \\
\text { Mean cost in US\$ } \\
{[95 \% \mathrm{Cl}]}\end{array}$ & $\begin{array}{l}\text { Health Center } \\
\text { Mean cost in US\$ } \\
{[95 \% \mathrm{Cl}]}\end{array}$ \\
\hline Consultation costs & 3.5 & 1.5 \\
& {$[2.8-4.2]$} & {$[0.3-1.8]$} \\
Medication costs & 4.6 & 4.4 \\
& {$[3.8-5.3]$} & {$[3.4-5.4]$} \\
Laboratory costs & 23.6 & 0.8 \\
& {$[16.9-30.3]$} & $0.2[0.5-1.2]$ \\
Transport costs & 1.2 & 0.7 \\
& {$[1.0-1.5]$} & {$[0.5-0.9]$} \\
Food costs & 1.4 & 0.2 \\
& {$[1.0-1.7]$} & {$[0.1-0.4]$} \\
Total costs & 34.2 & 7.7 \\
& {$[27.6-40.9]$} & {$[6.6-8.8]$} \\
\hline
\end{tabular}

Cl Confidence interval

The presence of co-morbidity was an independent predictor of uncontrolled HTN. Studies have shown that hypertensive patients with co-morbidity have poorer blood pressure control than those without co-morbidity [28].Given this high risk, patients with co-morbidities should not be managed by primary-level care facilities that only offer a minimum package of activities/treatment $[20,29]$. These results also suggest the need for guidelines on the management of HTN at the HC-level that include a global assessment of cardiovascular risk.

In contrast to other studies showing that the use of more than one antihypertensive drug is an important factor in HTN control, we found that patients on multiple antihypertensive drugs were more likely to have uncontrolled HTN than those on one drug [30, 31]. The use of more than one antihypertensive drug may mean that the patient has complicated HTN, which is difficult to control. Further, patients on multiple medications may have lower adherence [32].

Findings should be interpreted in light of the study's limitations. First, some of the data were based on selfreports, which are subject to bias. Second, we were unable to consider additional cost components such as productivity and opportunity costs. However, our overall findings corroborated the findings of previous studies and provide useful insights on how task shifting can affect the management of HTN.

\section{Conclusions}

This study shows that the management of HTN at primary healthcare level might be just as effective as at secondary level. However, the high proportion of patients with uncontrolled HTN underscores the need for HTN management guidelines at all healthcare levels. 


\section{Abbreviations}

DBP: Diastolic Blood Pressure; DRC: Democratic Republic of Congo; GRH: General Referral Hospital; HC: Health Center; HTN: Hypertension; KPHC: Kinshasa Primary Health-Care Network; LMICs: Low- and middleincome countries; NCDs: Non-communicable diseases; OR: Odds ratio; PHC: Primary Health Care; SBP: Systolic Blood Pressure; WHO: World Health Organization

\section{Acknowledgements}

We acknowledge all health care providers and patients for their participation. The authors acknowledge support from the African Doctoral Dissertation Research Fellowship (ADDRF) Program that is managed by the Africa Population and Health Research Center (APHRC) in partnership with the International Development Research Centre (IDRC).

\section{Funding}

The data collection and analysis time were funded through small grants to Kinshasa School of Public Health by the Future Health Systems (FHS) through the Africa Hub, which is funded by the Department for International Development (DFID). Publication was funded by the International Development Research Center (Grant Number 107508-001) and the John D. and Catherine T. MacArthur Foundation (Grant Number 14-107495-000-INP).

\section{Availability of data and materials}

The data are available from the corresponding author on reasonable request.

\section{About this supplement}

This article has been published as part of BMC Health Services Research Volume 17 Supplement 2, 2017: Research for health systems strengthening in Africa: studies by fellows of the African Doctoral Dissertation Research Fellowship (ADDRF) program. The full contents of the supplement are available online at https://bmchealthservres.biomedcentral.com/articles/ supplements/volume-17-supplement-2.

\section{Authors' contributions}

$J M, A L, D K$ and $Y C$ conceptualized the research; $A L$ and JM conducted the fieldwork and analyzed data; AL and EM wrote the paper. SA and MM revised the paper. $A L$ had the primary responsibility for the final content. All authors reviewed and approved the final manuscript.

\section{Ethics approval and consent to participate}

The study protocol was reviewed and approved by the institutional review board of the Kinshasa School of Public Health. All study participants provided written informed consent.

\section{Consent for publication}

Not applicable.

\section{Competing interests}

The authors declare that they have no competing interests.

\section{Publisher's Note}

Springer Nature remains neutral with regard to jurisdictional claims in published maps and institutional affiliations.

\footnotetext{
Author details

${ }^{1}$ Kinshasa School of Public Health, Faculty of Medicine, University of Kinshasa, Kinshasa, Democratic Republic of the Congo. ${ }^{2}$ Faculty of Economics and Management (FASEG), University of Lomé, Lomé, Togo. ${ }^{3}$ Programme de santé en milieu rural (SANRU), Kongo-central, Matadi, Democratic Republic of the Congo. ${ }^{4}$ School of Public Health, Université libre de Bruxelles, Brussels, Belgium.
}

\section{Published: 4 December 2017}

\section{References}

1. Lozano R, Naghavi M, Foreman K, Lim S, Shibuya K, Aboyans V, et al. Global and regional mortality from 235 causes of death for 20 age groups in 1990 and 2010: a systematic analysis for the global burden of disease study 2010 Lancet. 2012;380:2095-128.
2. World Health Organization. Global status report on non-communicable diseases. 2010. Geneva: World Health Organization; 2010.

3. World Health Organization. A global brief on hypertension, silent killer, global public health crisis 2013. Geneva: World Health Organization; 2013.

4. Kearney MP, Whelton M, Reynolds K, Muntner P, Whelton KP, He J. Global burden of hypertension: analysis of worldwide data. Lancet. 2005;365:217-23

5. Africaine U. Etat de l'hypertension en Afrique. Addis Ababa, Ethiopie: Conférence des Ministres de la santé de l'UA; 2013.

6. World Health Organization. World health statistics 2015. Available at: http:// www.who.int/mediacentre/news/releases/2015/world-health-statistics-2015/ fr/. Accessed 23 Aug 2017.

7. Njelekela MA, Mpembeni R, Mligiliche NL, Spiegelman D, Hertzmark E, Liu E, et al. Gender related differences in the prevalence of cardiovascular disease risk factors and their correlated in urban Tanzania. BMC Cardiovasc Disord. 2009;17:9-30.

8. Belue R, Okoror TA, Iwelunmor J, Taylor KD, Degboe AN, Agyemang C, et al. An overview of cardiovascular risk factor burden in sub-Saharan African countries: a socio-cultural perspective. Glob Health. 2009:22:5-10.

9. Mamo Y, Seid E, Adams S, Gardiner A, Parry E. A primary health care approach to the management of chronic diseases in Ethiopia: an example for other countries. Clin Med. 2007;7:228-31.

10. Alleyne G, Hancock C, Hughes P. Chronic and non-communicable diseases: a critical challenge for nurses globally. Int Nurs Rev. 2011;58:328-31.

11. Beaglehole R, Epping-Jordan J, Patel V, Chopra M, Ebrahim S, Kidd M, Haines A. Improving the prevention and management of chronic disease in lowincome and middle-income countries: a priority for primary health care. Lancet. 2008:372:940-9.

12. OMS. Statistiques Sanitaires Mondiales: Personnel de santé, infrastructures sanitaires et médicaments essentiels. Genève: OMS; 2009.

13. OMS. Optimisation des rôles du personnel de santé par la délégation des tâches pour améliorer l'accès aux interventions de santé maternelle et néonatale. Genève: OMS; 2013.

14. Chatora RR, Tumusime P. Primary health care: a review of its implementation in sub-Saharan Africa. Prim Health Care Res Dev. 2004;5: 296-306.

15. Lekoubou A, Awah P, Fezeu L, Sobngwi E, Kengne AP. Hypertension, diabetes mellitus and task shifting and their management in sub-Saharan Africa. Int J Environ Res Public Health. 2010;7:353-63.

16. Ogedegbe G, Gyamfi J, Plange-Rhule J, Surkis A, Rosenthal MD, Airhihenbuwa C, et al. Task shifting interventions for cardiovascular risk reduction in low-income and middle-income countries: a systematic review of randomized controlled trials. BMJ Open. 2014;4:e005983. https://doi.org/ 10.1196/bmjopen-2014-005983.

17. Mendis S, Johnston SC, Fan W, Oladapo O, Cameron A, Faramawi FM. Cardiovascular risk management and its impact on hypertension control in primary care in low-resource settings: a cluster randomized trial. Bull World Health Organ. 2010;88:412-9.

18. Adeyemo A, Tayo BO, Luke A, Ogedegbe O, Durazo-Arvizu R, Cooper RS The Nigerian-antihypertensive adherence trial (NA-HAT): a communitybased randomized trial. J Hypertens. 2013;31(1):201-7.

19. Ministère de la Santé Publique/RD Congo. Normes de zones de santé 2ième éd. Kinshasa: Ministère de la Santé Publique/ RD Congo; 2010.

20. Lulebo MA, Mutombo BP, Mapatano MA, Mafuta ME, Kayembe KP, Ntumba $\mathrm{TL}$, et al. Predictors of non-adherence to antihypertensive medication in Kinshasa, Democratic Republic of Congo: a cross-sectional study. BMC Res Notes. 2015;8:526

21. Kapongo YR, Lulebo MA, Mafuta ME, Mutombo BP, Dimbelolo MJC, Bieleli IE. Assessment of health service delivery capacities, health providers' knowledge and practices related to type 2 diabetes care in Kinshasa primary health care network facilities, Democratic Republic of Congo. BMC Health Serv Res. 2015;15:9.

22. Lemogoum D, Seedat YK, Mabadeje AF, Mendis S, Bovet P, Onwubere B, et al. Recommendations for prevention, diagnosis and management of hypertension and cardiovascular risk factors in sub-Saharan Africa. J Hypertens. 2003;21:1993-2000.

23. Morisky DE, Green LW, Levine DM. Concurrent and predictive validity of a self-reported measure of medication adherence. Med Care. 1986;24:67-74.

24. Shea S, Misera D, Ehrlich MA. Correlates of non adherence to hypertension treatment in an inner-city minority population. Am J Public Health. 1992;82: 1607-12. 
25. Patel RP, Taylor SD. Factors affecting medication adherence in hypertensive patient. Ann Pharmacother. 2002;36:40-5.

26. Lwelunmor J, Airhihenbuwa CO, Cooper R, Tayo B, Plange-Rhule J, Adanu R, et al. Prevalence, determinants and systems-thinking approaches to optimal hypertension control in West Africa. Glob Health. 2014;10:42.

27. Fahey T, Shroeder K, Ebrahim S. Educational and organizational interventions used to improve the management of hypertension in primary care: a systematic review. Br J Gen Pract. 2005:55:875-82.

28. OMS. Prévention des maladies cardiovasculaires: Guide de poche pour l'évaluation et la prise en charge du risque cardiovasculaire. Genève: Organisation Mondiale de la Santé; 2007.

29. Chia CY, Ching MS. Prevalence and predictors resistant hypertension in a primary care setting: a cross sectional study. BMC Fam Pract. 2014;15:131.

30. Vanderberg N, Meinke-Franze C, Fiss T, Baumeister SE, Hoffman W. Prevalence and determinants of controlled hypertension in a German population cohort. BMC Public Health. 2013;13:594.

31. Dennison CR, Peer N, Steyn K, Levitt NS, Hill MN. Determinants of hypertension care and control among peri-urban black south Africans: the HIHI study. Ethn Dis. 2007;17(3):484-91.

32. Laliberté F, Bookhart BK, Nelson WW, Lefebvre P, Schein JR, Leclaire JR, Duh MS. Impact of once-daily versus twice-daily dosing frequency on adherence to chronic medications among patients with venous Thromboembolism. Patient. 2013;6:213-24.

\section{Submit your next manuscript to BioMed Central} and we will help you at every step:

- We accept pre-submission inquiries

- Our selector tool helps you to find the most relevant journal

- We provide round the clock customer support

- Convenient online submission

- Thorough peer review

- Inclusion in PubMed and all major indexing services

- Maximum visibility for your research

Submit your manuscript at www.biomedcentral.com/submit

) Biomed Central 